\author{
Jamie M. Strachan \\ Michael P. J. DeVile
}

\section{Contrast-induced acute kidney injury: what is the prevalence of prevention protocols?}

Accepted: 5 January 2012

Published online: 1 February 2012

(C) Copyright jointly held by Springer and ESICM 2012

\section{Dear Editor,}

We read with interest the study by

Hoste and colleagues [2] and the accompanying editorial by Joannidis and Wiedermann [1]. Hoste has shown the incidence of contrastinduced acute kidney injury (CI-AKI) to be much higher in the critically ill patient and associated with a threefold increase in mortality. Those most at risk of renal injury appear to be patients with chronic renal disease, especially if they are hypotensive or on vasopressor therapy. The currently recommended treatment to optimise peri-procedural renal function is crystalloid volume loading. However, there is no consensus on which fluid to use and in what quantity - a state of affairs that could leave some clinicians sceptical as to whether an intervention is worthwhile at all. To assess current levels of practice in the UK, we performed an electronic survey to estimate the use of protocols and preventative therapies.
We directed the survey at all UK intensive care units (ICUs), as defined by the most recent UK Critical Care Directory (2008), via the Intensive Care Society website and by emailing individual hospitals.

We received responses from 117 individual ICUs from a possible 329 - a 36\% response rate. From these, $32(27.4 \%)$ claimed to have a protocol while $85(72.6 \%)$ did not. Protocols were more likely to have been implemented in cardiac or neuroscience ICUs (10/17 units; 58.8\%) than in teaching hospital ICUs $(8 / 32$ units; $25 \%$ ) or district general ICUs (14/68 units; $20.6 \%)$. Of the 32 units that claimed to have a protocol, 18 $(56 \%)$ used $N$-acetylcysteine (NAC) in a variety of dosing regimens, and the remaining $14(44 \%)$ did not use NAC. In units with a protocol, $1.26 \%$ sodium bicarbonate was the preferred fluid type (11 units; 34\%), followed by Hartmann's solution (10 units; $31 \%$ ) in a wide variety of volumes and regimen timings. Only five units $(16 \%)$ with protocols did not use fluid boluses. Of the 85 units that did not have a protocol, no consensus on the available evidence was stated as the reason by 48 units $(41 \%)$, while 20 units $(17 \%)$ felt this is a decision taken by radiology. Of these units without formal protocols, $17(20 \%)$ still gave NAC, whereas $68(80 \%)$ did not. A fluid regimen was used by 39 units $(46 \%)$, while $46(54 \%)$ did not give fluid boluses at all. Themes from free text comments included the feeling that the evidence is not yet convincing for the widespread use of standardised preventative measures as well as that taking a "risk benefit" approach, rather than using specific creatinine cut-off values, ought to determine contrast use.

Protocols for the prevention of CIAKI are not commonplace on ICUs in the UK. However, it appears that many clinicians are thinking about CI-AKI and freely prescribing some form of prophylaxis. We think this adds weight to Joannidis and Wiedermann's claim that more evidence, ideally in the form of a randomised control trial, is needed to convince the majority of ICU physicians that to follow a standardised approach in order to prevent renal injury from contrast media is worthwhile.

\section{References}

1. Joannidis M, Wiedermann CJ (2011) Radiocontrast-induced acute kidney injury in the ICU: worse than presumed? Intensive Care Med 37:1904-1906. doi:10.1007/s00134-011-2393-z

2. Hoste EA, Doom S, De Waele J, Delrue LJ, Defreyne L, Benoit DD, Decruyenaere J (2011) Epidemiology of contrast-associated acute kidney injury in ICU patients: a retrospective cohort analysis. Intensive Care Med 37:1921-1931. doi: 10.1007/s00134-011-2389-8

J. M. Strachan (

Intensive Care Unit, Royal Berkshire Hospital, Reading, RG1 5AN, UK e-mail: Strachan.jamie@gmail.com Tel.: +44-1183-227257

Fax: +44-1183-227256

M. P. J. DeVile

Nuffield Department of Anaesthetics, John Radcliffe Hospital,

Oxford, OX3 9DU, UK 